\title{
SWMPr: An R Package for Retrieving, Organizing, and Analyzing Environmental Data for Estuaries
}

by Marcus W Beck

\begin{abstract}
The System-Wide Monitoring Program (SWMP) was implemented in 1995 by the US National Estuarine Research Reserve System. This program has provided two decades of continuous monitoring data at over 140 fixed stations in 28 estuaries. However, the increasing quantity of data provided by the monitoring network has complicated broad-scale comparisons between systems and, in some cases, prevented simple trend analysis of water quality parameters at individual sites. This article describes the SWMPr package that provides several functions that facilitate data retrieval, organization, and analysis of time series data in the reserve estuaries. Previously unavailable functions for estuaries are also provided to estimate rates of ecosystem metabolism using the open-water method. The SWMPr package has facilitated a cross-reserve comparison of water quality trends and links quantitative information with analysis tools that have use for more generic applications to environmental time series.
\end{abstract}

\section{Introduction}

The development of low-cost, automated sensors that collect data in near real time has enabled a proliferation of standardized environmental monitoring programs (Glasgow et al., 2004; Fries et al., 2008). An invaluable source of monitoring data for coastal regions in the United States is provided by the National Estuarine Research Reserve System (NERRS, http://www. nerrs. noaa.gov/). This network of 28 estuary reserves was created to address long-term research, monitoring, education, and stewardship goals in support of coastal management. The System-Wide Monitoring Program (SWMP) was implemented in 1995 at over 140 stations across the reserves to provide a robust, longterm monitoring system for water quality, weather, and land-use/habitat change. Environmental researchers have expressed a need for quantitative analysis tools to evaluate trends in water quality time series given the quantity and quality of data provided by SWMP (System-Wide Monitoring Program Data Analysis Training, 2014).

This article describes the SWMPr package that was developed for estuary monitoring data from the SWMP. Functions provided by SWMPr address many common issues working with large datasets created from automated sensor networks, such as data pre-processing to remove unwanted information, combining data from different sources, and exploratory analyses to identify parameters of interest. Additionally, web applications derived from SWMPr and shiny illustrate potential applications using the functions in this package. The software is provided specifically for use with NERRS data, although many of the applications are relevant for addressing common challenges working with large environmental datasets.

\section{Overview of the SWMP network}

The SWMPr package was developed for the continuous abiotic monitoring network that represents a majority of SWMP data and, consequently, the most challenging to evaluate. Abiotic elements monitored at each reserve include water quality (water temperature, specific conductivity, salinity, dissolved oxygen concentration, dissolved oxygen saturation, depth, $\mathrm{pH}$, turbidity, chlorophyll fluorescence), weather (air temperature, relative humidity, barometric pressure, wind speed, wind direction, photosynthetically active radiation, precipitation), and nutrient data (orthophosphate, ammonium, nitrite, nitrate, nitrite + nitrate, chlorophyll a). Each of the 28 estuary reserves has no fewer than four water quality stations and one weather station at fixed locations. Water quality and weather data are collected at 15 minute intervals, whereas nutrient data are collected monthly at each water quality station. Data are made available through the Centralized Data Management Office (CDMO) web portal (http://cdmo. baruch.sc. edu/), where quality assurance/quality control (QAQC) measures are used to screen the information for accuracy and reliability. The final data include timestamped observations with relevant QAQC flags.

At the time of writing, the CDMO web portal provides over 60 million water quality, weather, and nutrient records that have been authenticated through systematic QAQC procedures. Records for each station are identified by a seven or eight character name that specifies the reserve, station, and 
Table 1: Retrieval functions available from the SWMPr package. Full documentation for each function is in the help file (e.g., execute ?all_params for individual functions or help. search ('retrieve', package $=$ 'SWMPr') for all).

\begin{tabular}{ll}
\hline Function & \multicolumn{1}{c}{ Description } \\
\hline all_params & $\begin{array}{l}\text { Retrieve records starting with the most recent at a given sta- } \\
\text { tion, all parameters. Wrapper to exportAllParamsXMLNew } \\
\text { function on web services. } \\
\text { all_params_dtrng } \\
\text { Retrieve records of all parameters within a given date } \\
\text { range for a station. Optional argument for a single param- } \\
\text { eter. Wrapper to exportAllParamsDateRangeXMLNew. }\end{array}$ \\
import_local & $\begin{array}{l}\text { Import files from a local path. The files must be in a specific } \\
\text { format, such as those returned from the CDMO using the }\end{array}$ \\
single_param & $\begin{array}{l}\text { Retrieve records for a single parameter starting with } \\
\text { the most recent at a given station. Wrapper to }\end{array}$ \\
& $\begin{array}{l}\text { exportSingleParamXMLNew function on web services. } \\
\text { site_codes }\end{array}$ \\
Get metadata for all stations. Wrapper to \\
site_codes_ind & $\begin{array}{l}\text { Get metadata for all stations at a single site. Wrapper to } \\
\text { NERRFilterStationCodesXMLNew function on web services. }\end{array}$ \\
\hline
\end{tabular}

parameter type. For example, 'apaebwq' is the water quality identifier ('wq') for the East Bay station ('eb') at the Apalachicola reserve ('apa'). Similarly, a suffix of 'met' or 'nut' specifies the weather (meteorological) or nutrient stations. All reserve names, stations, and date ranges for each parameter type can be viewed on the CDMO website. Alternatively, the site_codes (all sites) or site_codes_ind (single site) functions provided by SWMPr can be used. As noted below, an IP address must be registered with CDMO before using the data retrieval functions in SWMPr. Web services are provided by CDMO for direct access to SWMP data through http requests, in addition to standard graphical user interface options for selecting data. The data retrieval functions in SWMPr are simple calls to the existing retrieval functions on $\mathrm{CDMO}$ web services, as explained below.

\section{Structure of the SWMPr package}

SWMPr functions are categorized by one of three steps in the data workflow: retrieving, organizing, and analyzing. Functions for retrieving are used to import the data into $\mathrm{R}$ as a "swmpr" object class. Functions for organizing and analyzing the data provide methods for working with a "swmpr" object. The following describes the package structure, beginning with the retrieval functions, a description of the "swmpr" object returned after retrieval, and, finally, the organizing and analyzing functions.

\section{Data retrieval}

SWMPr can import data into $\mathrm{R}$ through direct download from the CDMO or by importing local data that was previously downloaded (Table 1). The IP address for the computer making the request must be registered if the first approach is used (see CDMO website). The site_codes or site_codes_ind functions can be used to view site metadata.

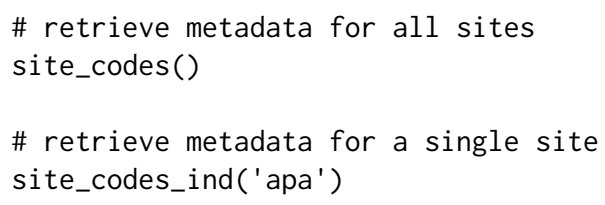

Retrieval functions to import data directly into R from the CDMO include all_params, all_params_dtrng, and single_param. Due to rate limitations on the CDMO server, the retrieval functions return a limited number of records with each request. However, the SWMPr functions use the native CDMO web services iteratively (i.e., within a loop) to obtain all requested records. Download time can be excessive for longer time series. 
\# all parameters for a station, most recent

all_params (' hudscwq')

\# get all parameters within a date range

all_params_dtrng('hudscwq', dtrng $=c\left({ }^{\prime 09 / 01 / 2013 ', ~ ' 10 / 01 / 2013 ')) ~}\right.$

\# get single parameter within a date range

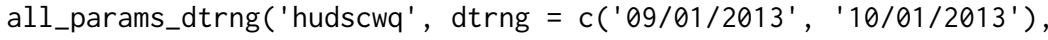

param = 'do_mgl')

\# single parameter for a station, most recent

single_param('hudscwq', param = 'do_mgl')

The second approach for data retrieval is to use the import_local function to import data into $\mathrm{R}$ after downloading from CDMO. This approach is most appropriate for large data requests. The import_local function is designed for data from the zip downloads feature in the advanced query section of the CDMO website. The zip downloads feature can be used to obtain a large number of records from multiple stations in one request. The downloaded data will be in a compressed folder that includes multiple .csv files by year for a given data type (e.g., apacpwq2002.csv, apacpwq2003.csv, apacpnut2002.csv, etc.). The import_local function can be used to import files directly from the zipped folder.

\section{The "swmpr" object class}

All data retrieval functions return a "swmpr" object that includes relevant data and several attributes describing the dataset. The data include a datetimestamp column in the timezone for a station and additional parameters for the data type (weather, nutrients, or water quality). Corresponding QAQC columns for each parameter are also returned if provided by the initial data request. The following shows an example of the raw data imported using all_params.

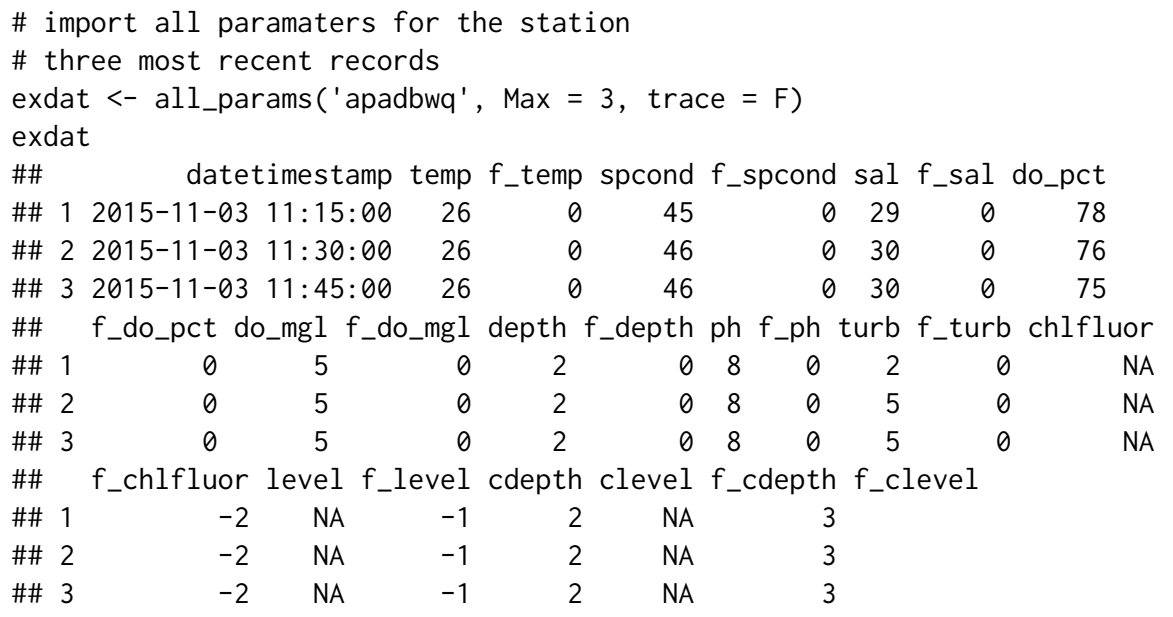

The attributes of a "swmpr" object are descriptors that are appended to the raw data (Table 2). These act as metadata that are used internally by many of the package functions and are updated as the data are processed. The attributes are not visible with the raw data but can be viewed as follows.

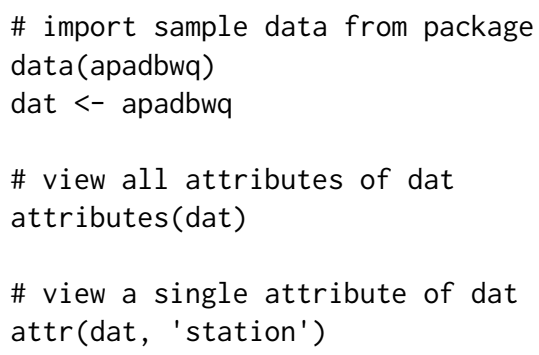

The "swmpr" object class was created for use with the organizing and analyzing functions. This uses the standard S3 object class system for R, such that specific methods for generic functions are developed for the object class. A "swmpr" object also secondarily inherits methods from the "data. frame" class. Available methods for the "swmpr" class are described below and can also be viewed: 
Table 2: Attributes of a "swmpr" object that describe characteristics of the data.

\begin{tabular}{|c|c|c|}
\hline Attributes & Class & Description \\
\hline names & character & $\begin{array}{l}\text { Column names of the entire data set, inherited from } \\
\text { the data. frame object class. }\end{array}$ \\
\hline row.names & integer & $\begin{array}{l}\text { Row names of the data set, inherited from the } \\
\text { data. frame object class. }\end{array}$ \\
\hline class & character & $\begin{array}{l}\text { Class of the data object indicating "swmpr" and } \\
\text { "data. frame". }\end{array}$ \\
\hline station & character & $\begin{array}{l}\text { Station identifier used by NERRS as a string with } 7 \text { or } \\
8 \text { characters. }\end{array}$ \\
\hline parameters & character & $\begin{array}{l}\text { Character vector of column names for data parameters, } \\
\text { e.g., ' do_mgl' , ' turb' , etc. }\end{array}$ \\
\hline qaqc_cols & logical & $\begin{array}{l}\text { Indicates if QAQC columns are present in the raw } \\
\text { data. }\end{array}$ \\
\hline date_rng & POSIXct & Start and end dates for the data. \\
\hline timezone & character & $\begin{array}{l}\text { Timezone of the station using the city/country } \\
\text { format }^{\mathrm{a}} \text {. }\end{array}$ \\
\hline stamp_class & character & $\begin{array}{l}\text { Class of the datetimestamp column, usually } \\
\text { "POSIXct" unless data have been aggregated. }\end{array}$ \\
\hline
\end{tabular}

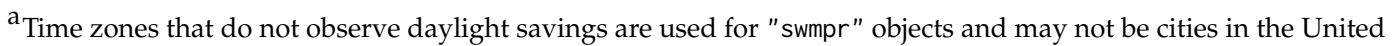
States. For example, America/Jamaica is used for Eastern Standard Time.

\# view available methods for swmpr class

methods (class $=$ 'swmpr')

\section{Data organizing}

The organize functions are used to 'clean' or prepare the imported data for analysis, including viewing and removal of QAQC flags, subsetting, combining replicate nutrient observations, creating a standardized time series, and combining data of different types (Table 3).

The qaqc function is a simple screen to retain observations from the data with specified QAQC flags (see http://cdmo.baruch.sc.edu/data/qaqc.cfm). Each parameter in the imported "swmpr" object will have a corresponding QAQC column of the same name with the added prefix $f_{-}$(e.g., f_do_mgl for do_mgl). Values in the QAQC column range from -5 to 5 to indicate the QAQC flag that was assigned by $\mathrm{CDMO}$ during initial processing. The qaqc function is used to remove observations in the raw data with given flags, with the default option to retain only values with the $\oslash$ QAQC flag (i.e., passed initial CDMO checks). Additionally, simple filters are used to remove obviously bad values (e.g., wind speed values less than zero or $\mathrm{pH}$ values greater than 12). Erroneous data entered as -99 are also removed. The function returns the original data with the QAQC columns removed and NA (not available) values for observations that do not meet the criteria specified in the function call.

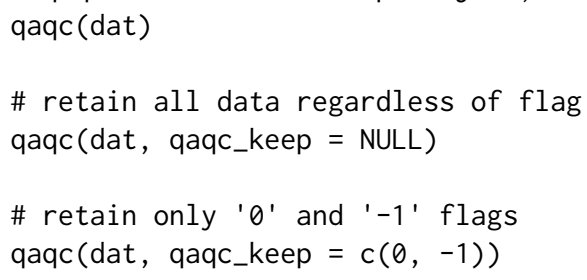

SWMP data often contain observations above or below the detection limit for the sensor or laboratory method used to quantify the parameters. For example, nutrient data exceeding the high sensor range are assigned a QAQC flag of -5 , whereas data below the low sensor range are assigned a QAQC flag of -4 . The presence of censored data is non-trivial and can influence the types of analyses that are appropriate for a time series (Helsel, 2012). A detailed discussion of methods for evaluating censored data is beyond the scope of the manuscript and existing methods for $\mathrm{R}$ are provided by other packages (e.g., cents, McLeod et al., 2014). However, the functions in SWMPr can be used to identify censored data based on the appropriate QAQC flag for a parameter. Viewing this information can be 
Table 3: Organizing functions available from the SWMPr package. Full documentation for each function is in the help file (e.g., execute ?comb for individual functions or help. search ('organize', package $=$ 'SWMPr') for all).

\begin{tabular}{|c|c|}
\hline Function & Description \\
\hline comb & $\begin{array}{l}\text { Combines "swmpr" objects to a common time series using } \\
\text { setstep, such as combining the weather, nutrients, and } \\
\text { water quality data for a single station. }\end{array}$ \\
\hline qaqc & $\begin{array}{l}\text { Remove QAQC columns and remove data based on QAQC } \\
\text { flag values for a "swmpr" object. }\end{array}$ \\
\hline qaqcchk & $\begin{array}{l}\text { View a summary of the number of observations in a } \\
\text { "swmpr" object that are assigned to each QAQC flag used } \\
\text { by CDMO. }\end{array}$ \\
\hline rem_reps & $\begin{array}{l}\text { Remove replicate nutrient data that occur on the same day. } \\
\text { The default is to average replicates. }\end{array}$ \\
\hline setstep & $\begin{array}{l}\text { Format data from a "swmpr" object to a continuous time } \\
\text { series at a given timestep. }\end{array}$ \\
\hline subset & $\begin{array}{l}\text { Subset by dates and/or columns for a "swmpr" object. This } \\
\text { is a method passed to the generic subset function in the } \\
\text { base installation. }\end{array}$ \\
\hline
\end{tabular}

helpful for determining how to further process the data with the qaqc function or alternative methods outside of SWMPr. The qaqcchk function returns a data. frame of the number of observations for a parameter that is assigned to all QAQC flags, including those for censored data. SWMP data should not be analyzed without viewing this information to determine an appropriate method to address data with questionable QAQC flags.

\# view the number of observations in each QAQC flag qaqcchk(dat)

A subset method added to the existing generic subset function in $\mathrm{R}$ is available for "swmpr" objects. This function is used to subset the data by date and/or a selected parameter. The date can be a single value or as two dates to select records within the range. The former case requires a binary operator as a character string passed to the operator argument, such as ' $>$ ' or ' $<=$ '. The subset argument for the date(s) must also be a character string of the format YYYY-mm-dd HH:MM for each element (e.g., ' 2007-01-01 06:30').

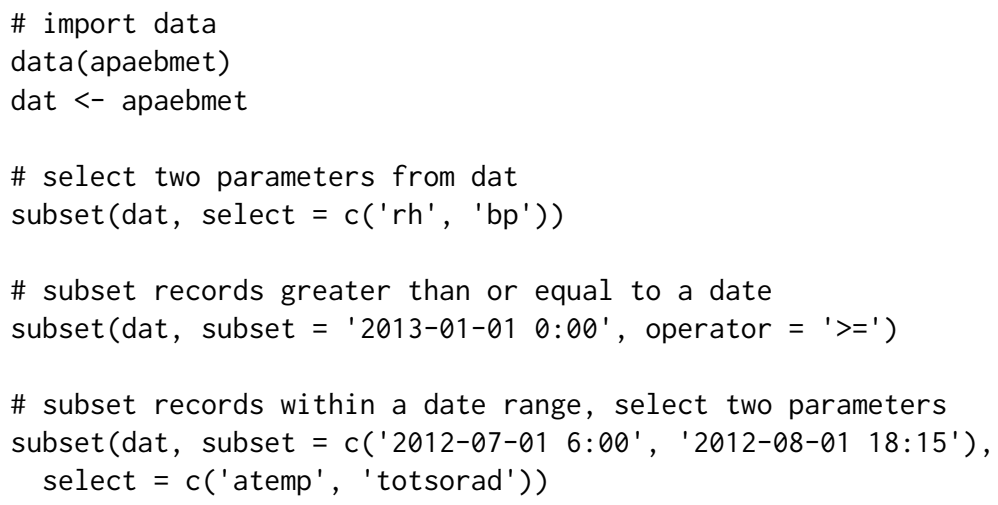

The setstep function formats a "swmpr" object to a continuous time series at a given time step. The function also has a default method making it useful for standardizing arbitrary time series to a given interval. The first argument of the function, timestep, specifies the desired time step in minutes starting from the nearest hour of the first observation. The second argument, differ, specifies the allowable tolerance in minutes for matching existing observations to the defined time steps in cases where the two are dissimilar. Values for differ that are greater than one half of the value of timestep are not allowed to prevent duplication of existing data. Likewise, the default value for differ is one half of the time step. 


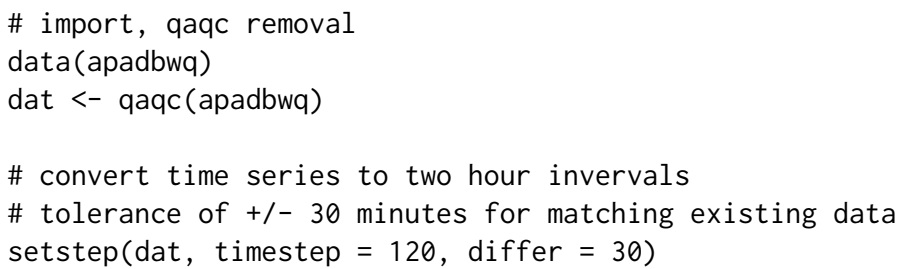

The comb function is used to combine multiple "swmpr" objects into a single object with a continuous time series at a given step. The setstep function is used internally such that timestep and differ are accepted arguments for comb. Data are combined by creating a master time series that is used to iteratively merge all "swmpr" objects. The time series for merging depends on the value passed to the method argument. Passing 'union' to method will create a time series that is continuous from the earliest and latest dates for all input objects, whereas 'intersect ' will create a continuous time series from the set of dates that are shared between input objects. A character string or numeric vector can also be used to specify which of the input objects to use as the master time series for combining. As with setstep, a default method for comb is provided to allow use with arbitrary data structures. Both functions treat missing data as NA values, either for observations that exceed the allowable tolerance for the differ argument of setstep or for portions of time series that do not overlap given the method argument passed to comb.

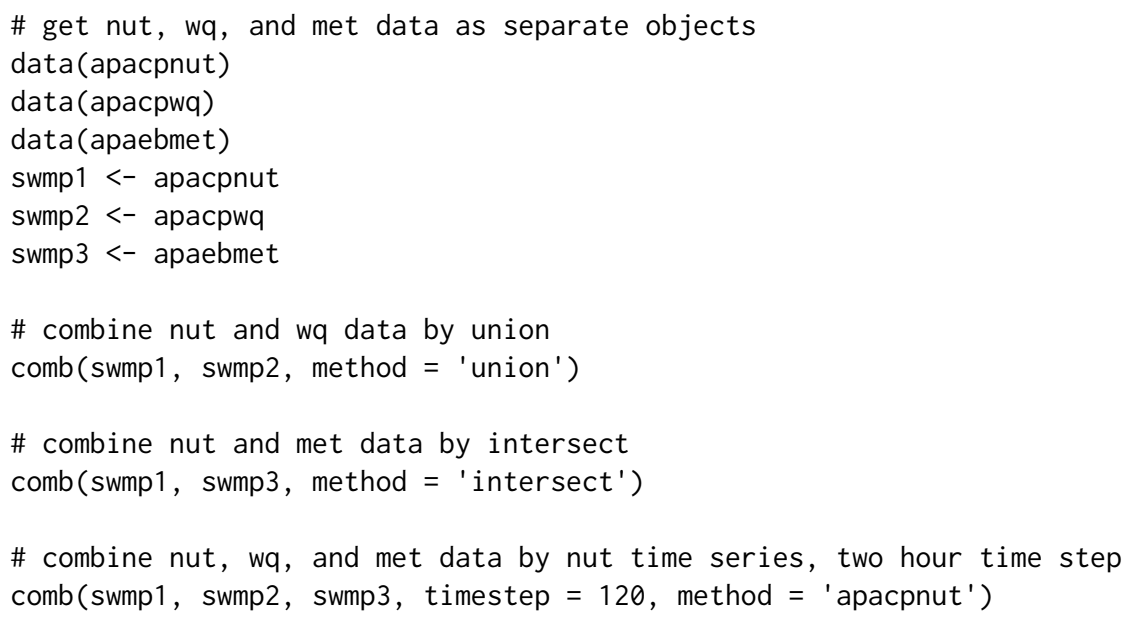

\section{Data analysis}

The analysis functions range from general purpose tools for time series analysis to more specific functions for working with continuous monitoring data in estuaries (Table 4). The general purpose tools are "swmpr" methods for existing S3 generics or are slight modifications to existing functions. These include aggreswmp to combine observations by set periods of time (e.g., weeks, months), smoother to average time series with a moving window, and approx to substitute missing data with interpolated values. For brevity, the general functions are not discussed. More specific functions for environmental time series include decomposition functions, decomp and decomp_c $j$, and functions to estimate and plot ecosystem metabolism from combined water quality and weather data. Several plotting methods to facilitate analysis are also descibed below.

The disaggregation of time series into additive or multiplicative components is a common application for trend analysis. The decomp function is a simple wrapper to decompose (Kendall and Stuart, 1983) that separates a time series into a trend, cyclical variation (e.g., daily or annual), and the remainder (Figure 1). An additive decomposition assumes that the cyclical component of the time series is stationary (i.e., the variance is constant); otherwise, a multiplicative decomposition can be used. The frequency argument describes the periodicity of the cyclical parameter in units of the native time step. For example, the frequency for a parameter with daily periodicity would be 96 if the time step is 15 minutes ( 24 hours * 60 minutes / 15 minutes). For simplicity, character strings of 'daily ' or ' annual' can be supplied in place of numeric values, although any number can be used to identify an arbitrary cyclical component. A starting value of the time series must be supplied in the latter case that indicates the sequence in the cycle for the first observation (see ts for details).

\# get data

data(apadbwq)

dat $<-$ apadbwq 
Table 4: Analysis functions available from the SWMPr package. Full documentation for each function is in the help file (e.g., execute ?aggreswmp for individual functions or help. search ('analyze', package $=$ 'SWMPr') for all).

\begin{tabular}{|c|c|}
\hline Function & Description \\
\hline aggreswmp & $\begin{array}{l}\text { Aggregate "swmpr" objects for different time periods - } \\
\text { years, quarters, months, weeks, days, or hours. The aggre- } \\
\text { gation function defaults to the mean. }\end{array}$ \\
\hline aggremetab & $\begin{array}{l}\text { Aggregate metabolism data from a "swmpr" object. This } \\
\text { is primarly used within plot_metab but may be useful for } \\
\text { simple summaries of daily metabolism data. }\end{array}$ \\
\hline ecometab & $\begin{array}{l}\text { Estimate ecosystem metabolism for a combined water qual- } \\
\text { ity and weather dataset using the open-water method } \\
\text { (Odum, 1956). }\end{array}$ \\
\hline decomp & $\begin{array}{l}\text { Decompose a "swmpr" time series into trend, seasonal, } \\
\text { and residual components. This is a simple wrapper to } \\
\text { decompose (Kendall and Stuart, 1983). Decomposition of } \\
\text { monthly or daily trends is possible. }\end{array}$ \\
\hline decomp_cj & $\begin{array}{l}\text { Decompose a "swmpr" time series into grandmean, annual, } \\
\text { seasonal, and events components. This is a simple wrapper } \\
\text { to decompTs in the wq package (Jassby and Cloern, 2014). } \\
\text { Only monthly decomposition is possible. }\end{array}$ \\
\hline hist & Plot a histogram for a single variable. \\
\hline lines & Add lines to an existing plot created with plot. \\
\hline map_reserve & $\begin{array}{l}\text { Create a map of all stations in a reserve using the ggmap } \\
\text { package (Kahle and Wickham, 2013). }\end{array}$ \\
\hline na. approx & $\begin{array}{l}\text { Linearly interpolate missing data (NA values) in a "swmpr" } \\
\text { object. }\end{array}$ \\
\hline overplot & $\begin{array}{l}\text { Plot multiple time series in a "swmpr" object on the same } \\
\text { y-axis. }\end{array}$ \\
\hline plot & Plot a univariate time series for a "swmpr" object. \\
\hline plot_metab & $\begin{array}{l}\text { Plot ecosystem metabolism estimates after running } \\
\text { ecometab on a combined "swmpr" object. }\end{array}$ \\
\hline plot_summary & $\begin{array}{l}\text { Create summary plots of seasonal/annual trends and } \\
\text { anomalies for a single parameter. }\end{array}$ \\
\hline smoother & $\begin{array}{l}\text { Smooth "swmpr" objects with a moving window average, } \\
\text { passed to filter. }\end{array}$ \\
\hline
\end{tabular}


\# subset for daily decomposition

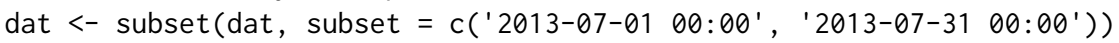

\# daily decomposition of DO and plot

dc_dat <- decomp (dat, param = 'do_mgl', frequency = 'daily')

plot (dc_dat)

\section{Decomposition of additive time series}

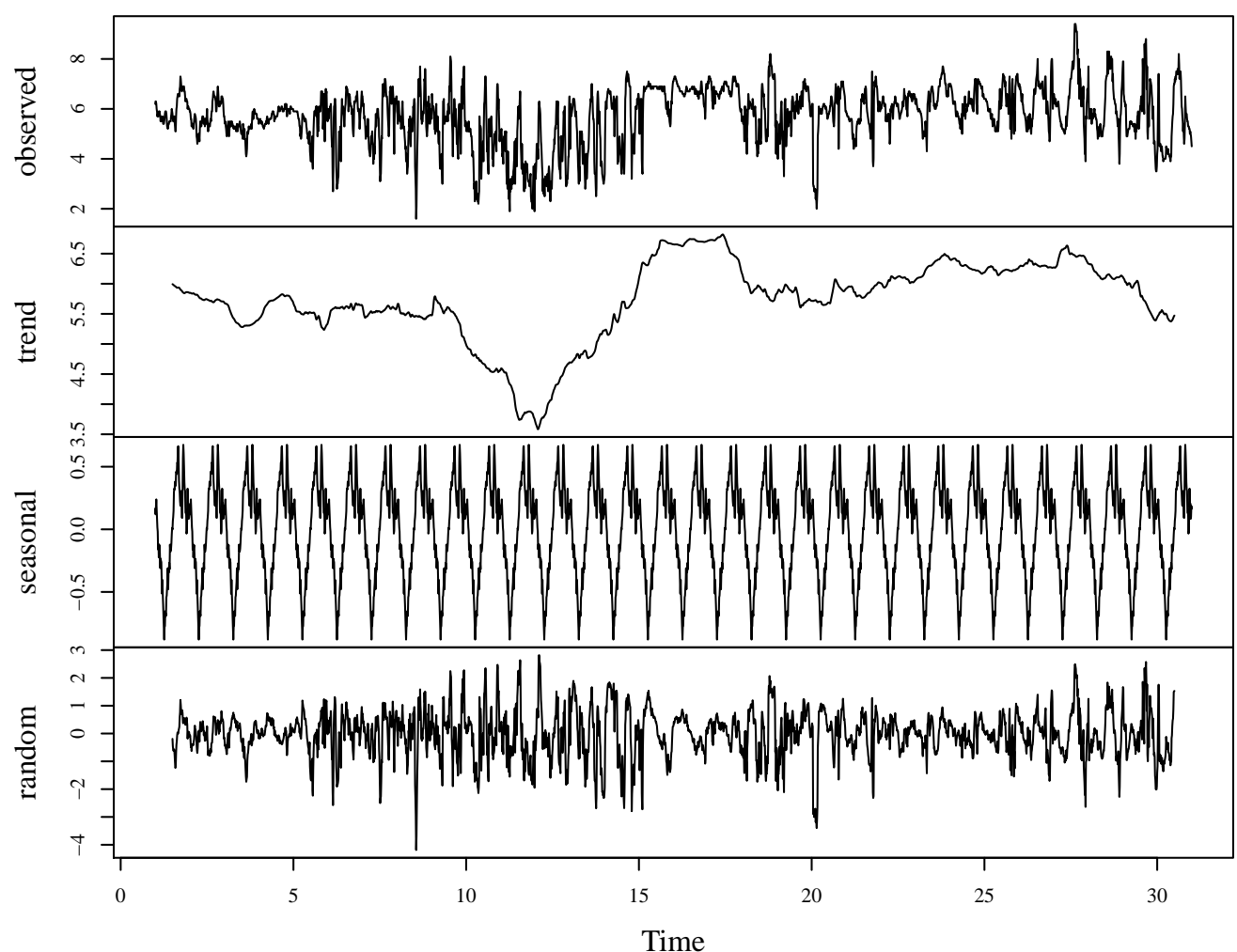

Figure 1: An additive decomposition of dissolved oxygen into a trend, seasonal (daily), and random component using the decomp function.

An alternative approach for decomposition is provided by the decomp_cj function, which is a simple wrapper to the decompTs function in the wq package (Cloern and Jassby, 2010; Jassby and Cloern, 2014). The decomp_cj function is a monthly decomposition for characterizing relatively longterm trends. This approach works best for nutrient data that are typically obtained on a monthly cycle. The time series is decomposed into the grandmean, annual, seasonal, and events components (Figure 2), as compared to trend, seasonal, and random components for the decomp function above. For both functions, the random or events components can be considered anomalies that do not follow the trends in the remaining categories. Additional arguments passed to decompTs can be used with decomp_cj, such as startyr, endyr, and type. Values passed to type are mult (default) or add, referring to multiplicative or additive decomposition.

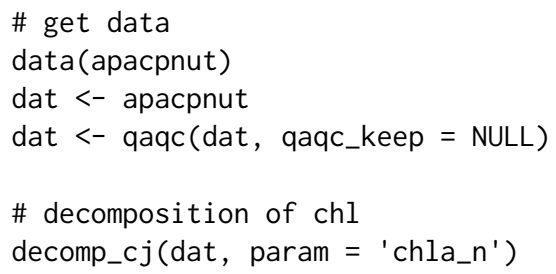

Estimates of ecosystem metabolism provide a measure of system productivity to evaluate whether an ecosystem is a net source or sink of organic material. The open-water method (Odum, 1956) is a 


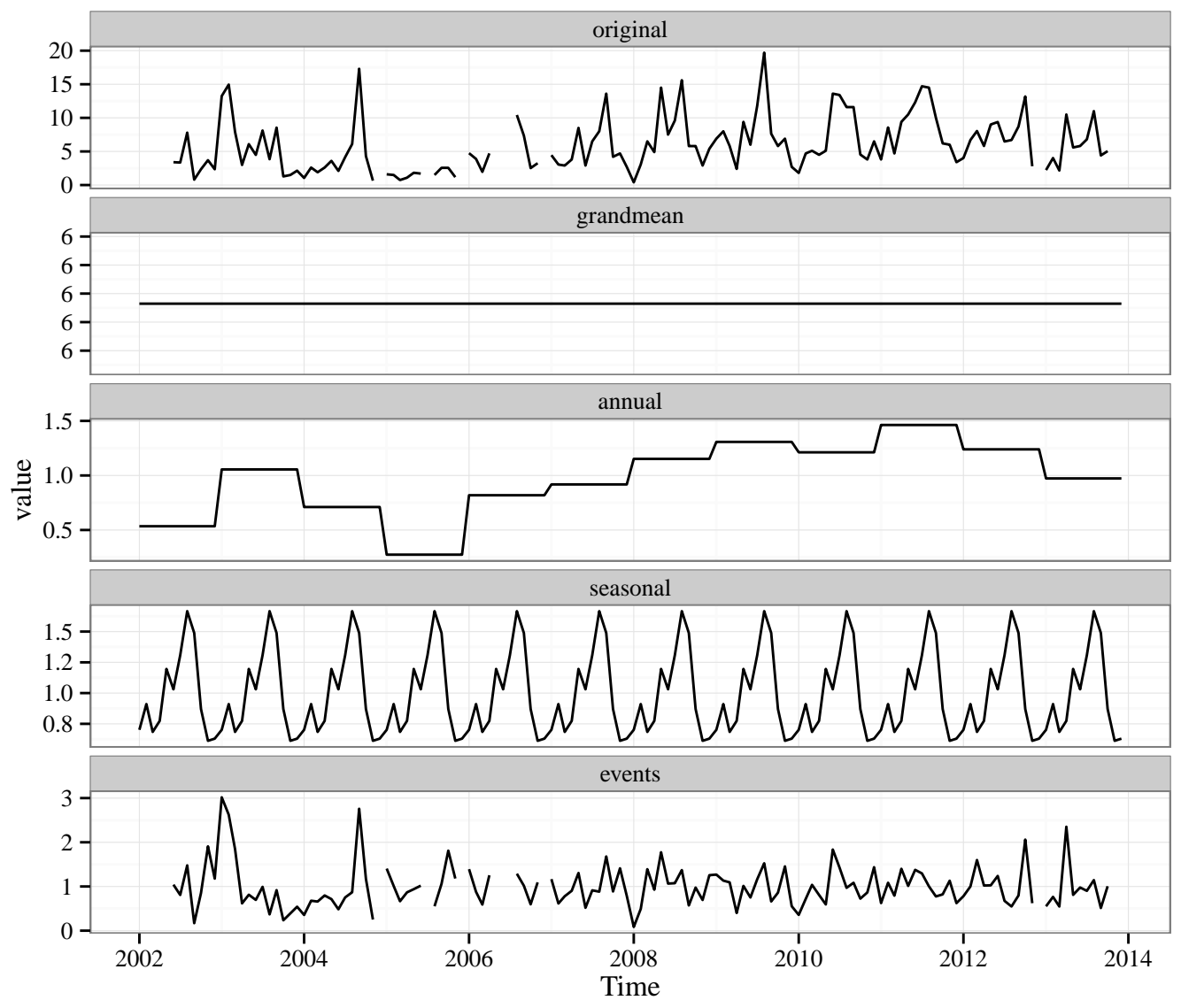

Figure 2: Additive decomposition of a multi-year chlorophyll time series into the grandmean, annual, seasonal, and events components using the decomp_cj function.

common approach to quantify metabolism using a mass balance equation that describes the change in dissolved oxygen over time from the balance between photosynthetic and respiration processes, corrected using an empirically constrained air-sea gas diffusion model (Ro and Hunt, 2006; Thébault et al., 2008). A detailed discussion of the method is beyond the scope of this article, although users are encouraged to consult references herein for additional information (see Kemp and Testa (2012); Needoba et al. (2012); Caffrey et al. (2013), also the package help files). Methods for estuaries have not previously been available in $\mathrm{R}$, although the StreamMetabolism package provides an approach for freshwater systems. The following is an example that shows use of ecometab with a combined water quality and weather data set. Monthly aggregations of the raw, daily estimates are plotted using plot_metab (Figure 3).

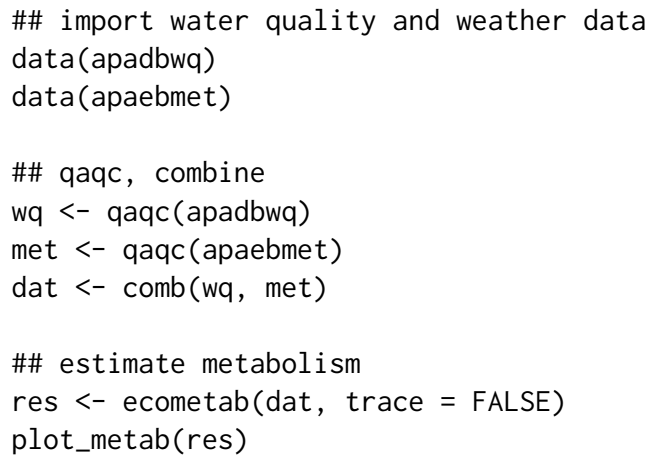

Exploratory graphics are also useful for evaluating general trends in observed data. Several graphics showing seasonal and annual trends for a single SWMP parameter can be obtained using the plot_summary function (Figure 4). The plots include monthly distributions, monthly anomalies, and annual anomalies in multiple formats. An interactive shiny web application (Chang et al., 2015) that uses this function is available for viewing results for all SWMP sites (see the Applications using the 


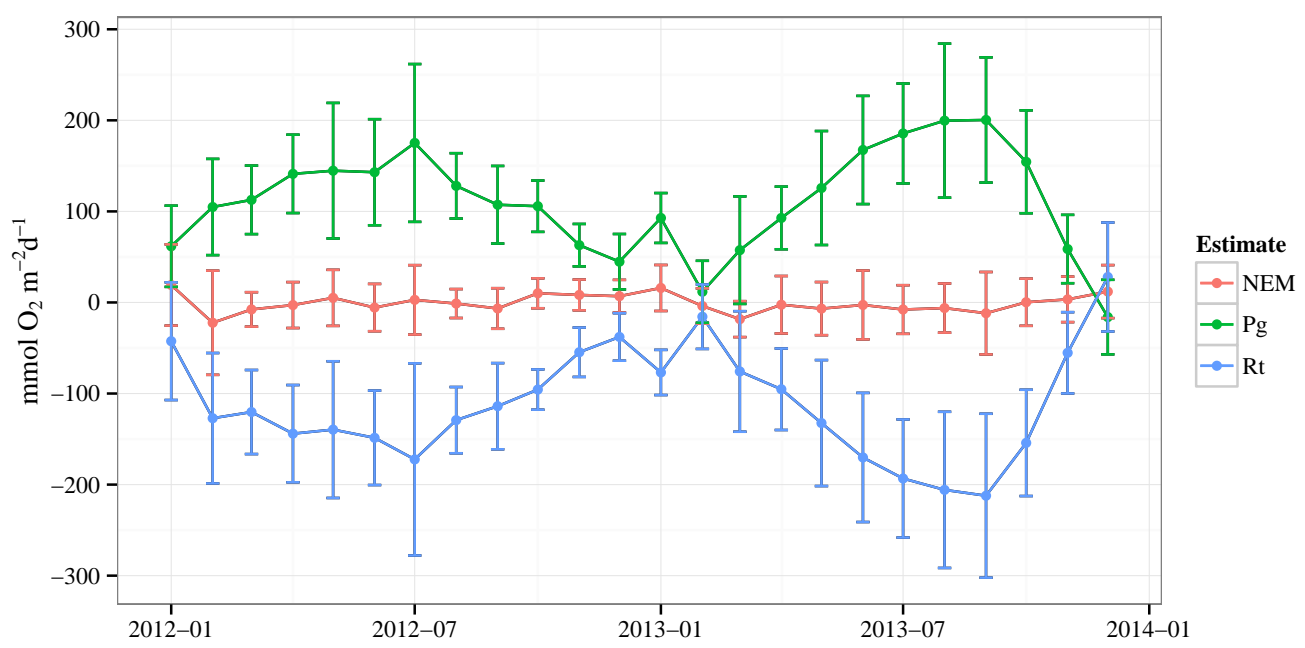

Figure 3: Monthly means (95\% confidence) of ecosystem metabolism estimates (net ecosystem metabolism, gross production, and total respiration) for combined water quality and weather data for two years at Apalachicola Bay, Florida.

SWMPr package section).

\#\# import data

data(apacpnut)

dat <- qaqc(apacpnut)

\#\# plot

plot_summary (dat, param $=$ 'chla_n', years $=c(2007,2013))$
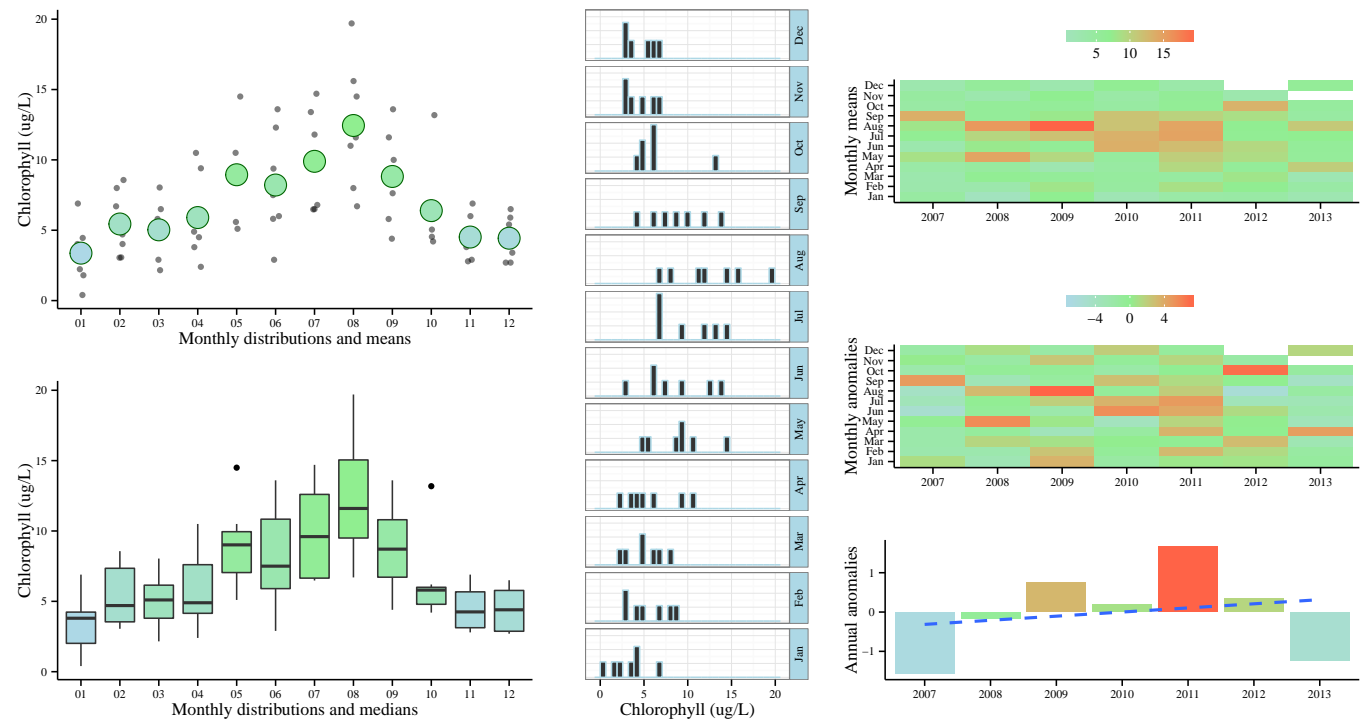

Chlorophyll (ug/L) ${ }^{15}$

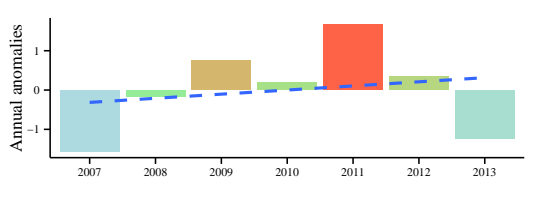

Figure 4: Summaries of a multi-year chlorophyll time series using the plot_summary function. Summaries include monthly distributions (means on top left, quantiles on bottom left), monthly histograms (center), monthly means by year (top right), deviation from monthly means (middle right), and annual trends as deviations from the grand mean (bottom right)

Similarly, the overplot function provides an alternative approach to viewing observed data from the same station. This function uses the base graphics package to plot multiple time series on the same $y$-axis (Figure 5).

\#\# import data

data (apacpwq) 

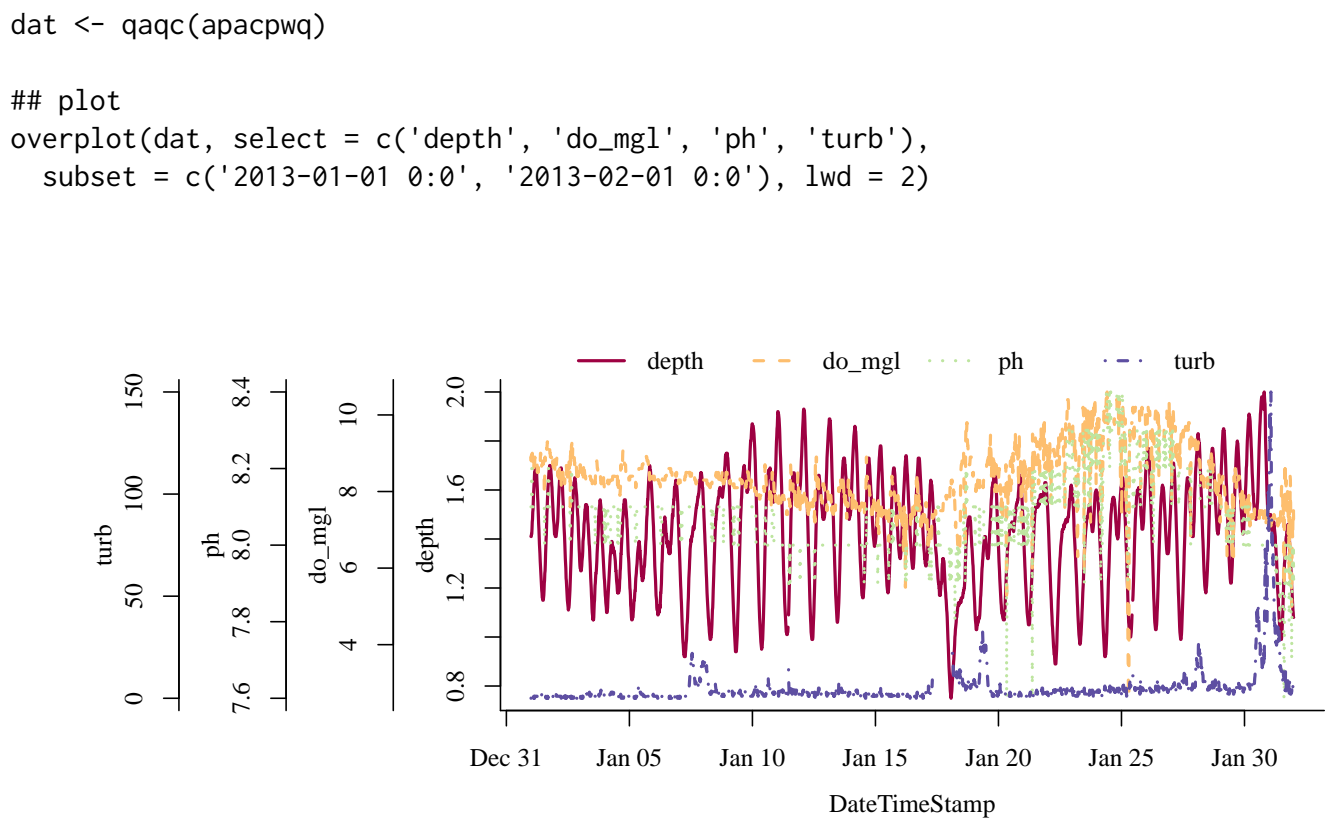

Figure 5: The overplot function plots multiple variables on the same y-axis.

Finally, the map_reserve function can be used to create a map of stations at a reserve using the ggmap package (Figure 6, Kahle and Wickham (2013)). The function uses Google maps of four types that can be set with the map_type argument: terrain (default), satellite, roadmap, or hybrid. The zoom argument can be chosen through trial and error depending on the spatial extent of the reserve.

\# plot the stations at Jacques Cousteau reserve map_reserve('jac')

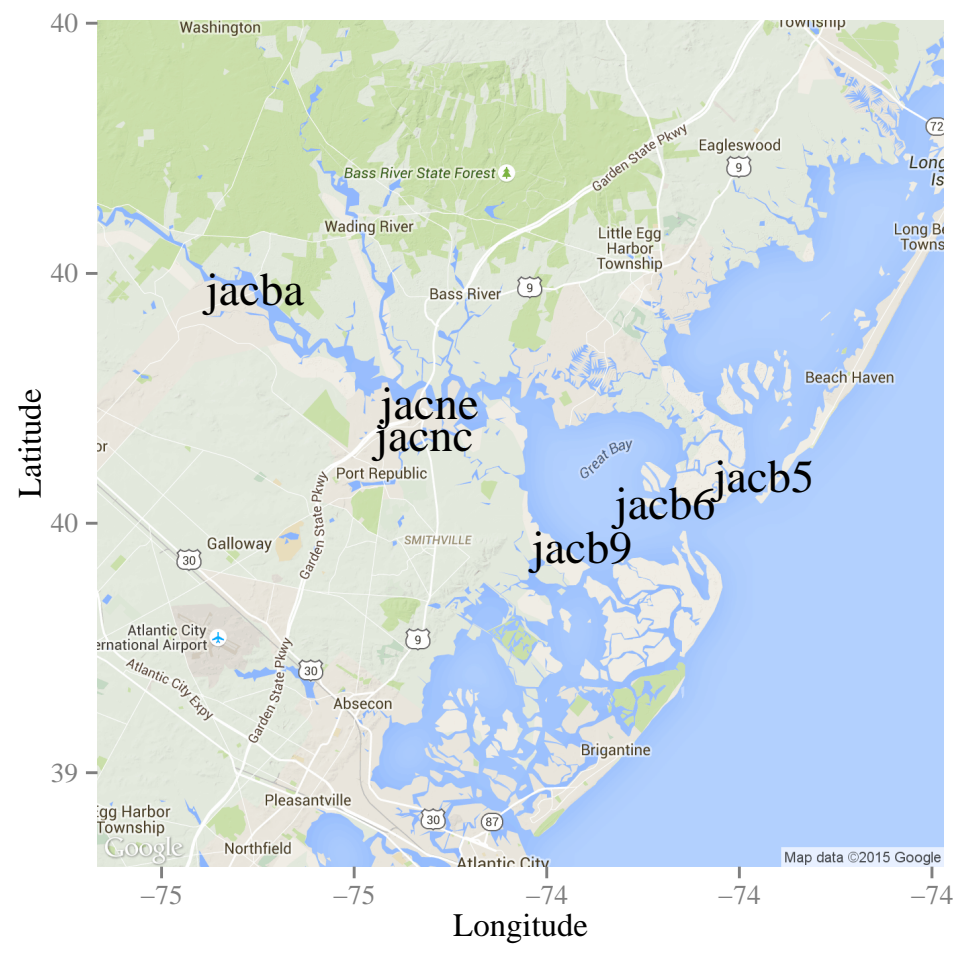

Figure 6: Locations of all sites at the Jacques Cousteau reserve using the map_reserve function. 


\section{Applications using the SWMPr package}

Two shiny web applications illustrate the improved ability to synthesize and evaluate multi-year time series using SWMPr. The first application evaluates trends in SWMP data within and between sites using an interactive leaflet map (Cheng and Xie (2015), Figure 7): https: / /beckmw. shinyapps. io / swmp_comp. Trends between reserves can be viewed using the map, whereas trends at individual sites can be viewed by clicking on a map location. Site-level trends are shown below the map with a simple linear regression to show an increase or decrease in values over time, whereas trends between sites are shown on the map for each station as circles that identify the direction and significance of the trend. More robust methods for evaluating trends are currently not provided by the application and the use of simple linear regression is meant for initial exploratory analysis. The second application provides graphical summaries of water quality, weather, or nutrient station data at individual stations using the plot_summary function: https://beckmw. shinyapps.io/swmp_summary/. The output is identical to Figure 4 with the addition of drop down menus to select the station, date range, and parameter for plotting.

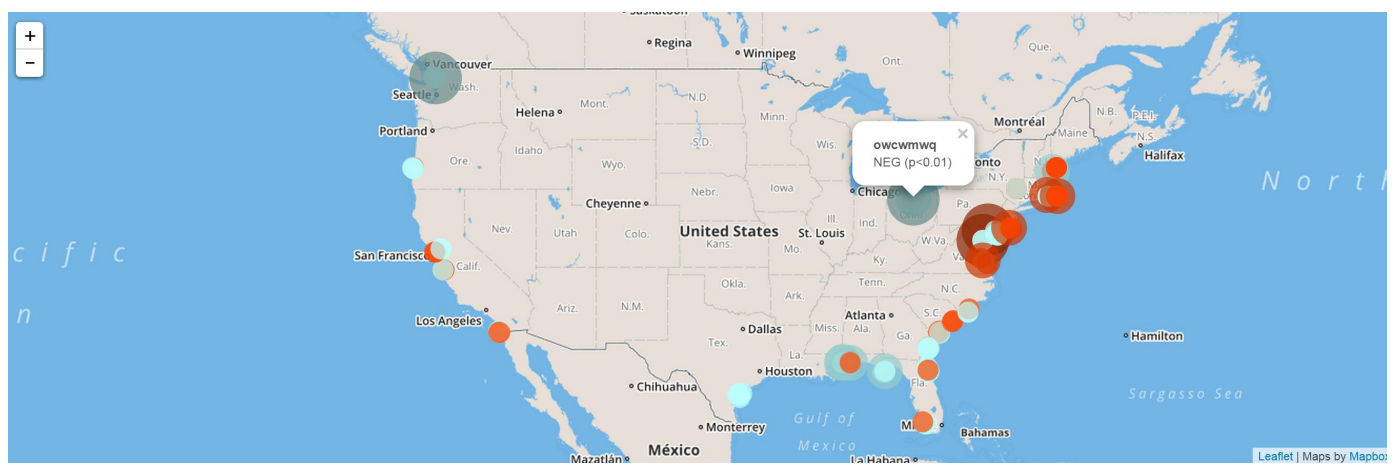

Trends in SWMP parameters

Created by Marcus W. Beck, beck.marcus@epa.gov, Todd O'Brien, todd.obrien@noaa.gov

This widget is an interactive tool to evaluate trends in SWMP data. Trends are described by an increase or decrease in values over time using a simple linear regression of summarized data. The regression for each station can be viewed by clicking on a map location. Trends at each station are plotted as circles that identify the direction and significance of the trend. The trend direction is blue for decreasing and red for increasing. The significance is indicated by radius of the circle and color shading where larger points with darkers colors indicate a strong trend. Original data are available from hitp:///cdmo.baruch.sc.edu/. See the Select parameter:
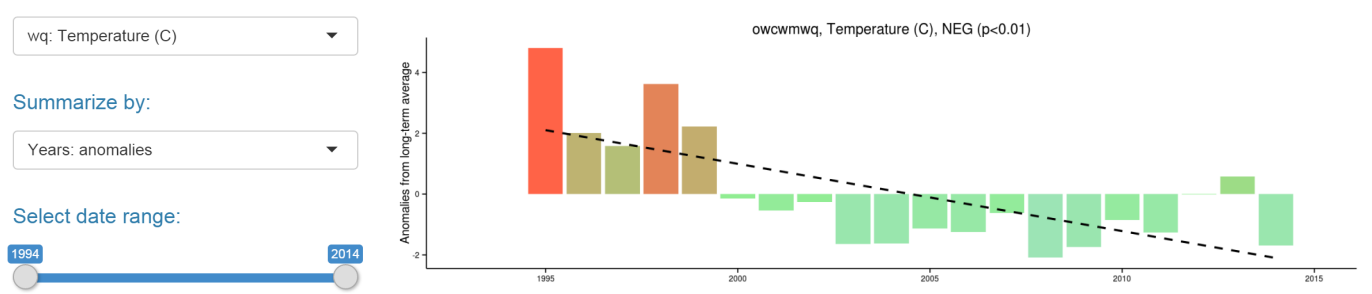

Figure 7: Online application for comparing trends in SWMP data parameters using an interactive map. Link: https://beckmw. shinyapps.io/swmp_comp

\section{Conclusions}

SWMPr was developed to augment existing data management programs (i.e., CDMO) by providing a bridge betwen the raw data and the analysis software through its numerous data retrieval functions (Table 1). Established QAQC methods and data processing techniques are also enhanced with SWMPr by functions that filter observations for different QAQC flags (qaqc) and subset by selected dates or variables (subset). Additionally, challenges comparing differents datasets are addressed by the setstep and comb functions that standardize and combine time series. Finally, the analysis functions provide numerous tools to implement common analyses for time series and more specific methods for water quality data. Further development of the package will include modifications and additional functions to better integrate data analysis with the quality of information provided by SWMP. Several functions include default methods to extend use beyond the "swmpr" object and additional development will continue to focus on modifying the package to handle arbitrary data structures. These challenges are not unique to the SWMP database such that many of the functions will facilitate evaluations of more generic time series datasets. 


\section{Acknowledgments}

I acknowledge the significant work of NERRS researchers and staff that has allowed access to highquality monitoring data. Thanks to Todd O'Brien for the inspiration for the online widgets. Thanks to Mike Murrell and Jim Hagy III for assistance with the ecosystem metabolism functions. Thanks to Jeff Hollister for providing useful comments on an earlier draft.

\section{Bibliography}

J. M. Caffrey, M. C. Murrell, K. S. Amacker, J. Harper, S. Phipps, and M. Woodrey. Seasonal and interannual patterns in primary production, respiration and net ecosystem metabolism in 3 estuaries in the northeast Gulf of Mexico. Estuaries and Coasts, 37(1):222-241, 2013. [p227]

W. Chang, J. Cheng, J. Allaire, Y. Xie, and J. McPherson. shiny: Web Application Framework for R, 2015. URL http://CRAN. R-project.org/package=shiny. R package version 0.11.1. [p227]

J. Cheng and Y. Xie. leaflet: Create Interactive Web Maps with the JavaScript 'Leaflet' Library, 2015. URL http://CRAN.R-project.org/package=leaflet. R package version 1.0.0. [p230]

J. E. Cloern and A. D. Jassby. Patterns and scales of phytoplankton variability in estuarine-coastal ecosystems. Estuaries and Coasts, 33(2):230-241, 2010. [p226]

D. P. Fries, S. Z. Ivanov, P. H. Bhanushali, J. A. Wilson, H. A. Broadbent, and A. C. Sanderson. Broadband, low-cost, coastal sensor nets. Oceanography, 20(4):150-155, 2008. [p219]

H. B. Glasgow, J. M. Burkholder, R. E. Reed, A. J. Lewitus, and J. E. Kleinman. Real-time remote monitoring of water quality: a review of current applications, and advancements in sensor, telemetry, and computing technologies. Journal of Experimental Marine Biology and Ecology, 300(1-2):409-448, 2004. [p219]

D. R. Helsel. Statistics for Censored Environmental Data Using Minitab and R. John Wiley \& Sons, Inc., Hoboken New Jersey, 2nd edition, 2012. [p222]

A. D. Jassby and J. E. Cloern. wq: Exploring water quality monitoring data, 2014. URL http: //CRAN. Rproject.org/package=wq. R package version $0.4-1$. [p225, 226]

D. Kahle and H. Wickham. ggmap: Spatial visualization with ggplot2. The R Journal, 5(1):144-161, 2013. URL http://journal.r-project.org/archive/2013-1/kahle-wickham. pdf. [p225, 229]

W. M. Kemp and J. M. Testa. Metabolic balance between ecosystem production and consumption. In E. Wolanski and D. S. McLusky, editors, Treatise on Estuarine and Coastal Science, pages 83-118. Academic Press, New York, 2012. [p227]

M. Kendall and A. Stuart. The Advanced Theory of Statistics, volume 3. MacMillan Publishing Company, New York, New York, 1983. [p224, 225]

A. McLeod, N. M. Mohammad, J. Veenstra, and A. El-Shaarawi. cents: Censored time series, 2014. URL http://CRAN.R-project. org/package=cents. R package version 0.1-41. [p222]

J. A. Needoba, T. D. Peterson, and K. S. Johnson. Method for the quantification of aquatic primary production and net ecosystem metabolism using in situ dissolved oxygen sensors. In S. M. TiquiaArashiro, editor, Molecular Biological Technologies for Ocean Sensing, pages 73-101. Springer, New York, 2012. [p227]

H. T. Odum. Primary production in flowing waters. Limnology and Oceanography, 1(2):102-117, 1956. [p225, 226]

K. S. Ro and P. G. Hunt. A new unified equation for wind-driven surficial oxygen transfer into stationary water bodies. Transactions of the American Society of Agricultural and Biological Engineers, 49 (5):1615-1622, 2006. [p227]

System-Wide Monitoring Program Data Analysis Training. SWMP data analysis training workshop provided at the 2014 NERRS/NERRA annual meeting, November 17, 2014. http: //copepod.org/ nerrs-swmp-workshop/. [p219]

J. Thébault, T. S. Schraga, J. E. Cloern, and E. G. Dunlavey. Primary production and carrying capacity of former salt ponds after reconnection to San Francisco Bay. Wetlands, 28(3):841-851, 2008. [p227] 
Marcus W Beck

US Environmental Protection Agency

National Health and Environmental Effects Research Laboratory

Gulf Ecology Division

1 Sabine Island Drive, Gulf Breeze, FL 32651

USA

beck.marcus@epa.gov 